\title{
The Time Course of Attentional Biases in Pain: A Meta-Analysis of Eye Tracking Studies
}

Emma Blaisdale Jones ${ }^{a}$, Louise Sharpe ${ }^{a}$, Sally Andrews ${ }^{a}$, Ben Colagiuria, Joanne Dudeney ${ }^{b}$, Elaine Fox ${ }^{c}$, Lauren C. Heathcote $^{d}$, Jennifer Y.F. Lau e, Jemma Todd ${ }^{a}$, Stefaan Van Damme ${ }^{f}$, Dimitri M.L. Van Ryckeghem $^{f, g, h}$, Tine Vervoort ${ }^{f}$

aSchool of Psychology, University of Sydney, NSW, 2006, Australia

${ }^{b}$ Department of Psychology, Macquarie University, Australia

'Department of Experimental Psychology, University of Oxford, United Kingdom

dDepartment of Anesthesiology, Perioperative, and Pain Medicine, Stanford University, United States

eDepartment of Psychology, King's College London, London, United Kingdom

fDepartment of Experimental Clinical and Health Psychology, Ghent University, Belgium

$\mathrm{g}$ Department of Behavioural and Cognitive Sciences, University of Luxembourg, Esch-sur-Alzette, Luxembourg

$\mathrm{h}$ Department of Clinical Psychological Science, Maastricht University, Maastricht, Netherlands

*Corresponding author. Address: Louise Sharpe, School of Psychology, 450 Brennan MacCallum Building (A18), University of Sydney, NSW, 2006, Australia

Tel: +61 0293514558 Fax: +61 0293517328

Email: louise.sharpe@sydney.edu.au

Number of pages: 42 Number of Figures: 1 Number of Tables: 3

Author Contribution: First (EJ) and second author (LS) were responsible for conception and design, data extraction, data analysis and interpretation and initial drafting of the manuscript. All other authors (SA, $B C, J D, E F, L H, J L, J T, S V D, D V R, T V$ ) contributed equally by reviewing and amending the protocol, completing a portion of title and abstract review or risk of bias assessment, and/or by reviewing the draft, responding to reviewer comments, and approving the final manuscript. These authors have been listed in alphabetical order.

Significance: This is the first meta-analysis in the pain literature looking specifically at eye-tracking methodology to measure attentional biases. Results indicate that when more direct and reliable measurements of attention (gaze behaviour) are used rather than reaction time indices, attentional biases to pain are ubiquitous and not specific to people with chronic pain. Future research should develop paradigms to better conceptualize the complex nature of attentional biases towards pain. 


\begin{abstract}
Previous meta-analyses investigating attentional biases towards pain have utilized reaction time measures. Eye-tracking methods have been adopted to more directly and reliably assess biases, but this literature has not been synthesized in relation to pain. This meta-analysis aimed to investigate the nature and time-course of attentional biases to pain-related stimuli in participants of all ages with and without chronic pain using eye-tracking studies; and determine the role of task parameters and theoretically relevant moderators. After screening, 24 studies were included with a total sample of 1425 participants. Between-group analyses revealed no significant overall group differences for people with and without chronic pain on biases to pain-related stimuli. Results indicated significant attentional biases towards pain related words or pictures across both groups on probability of first fixation $(k=21, g$ $=0.43,95 \% \mathrm{Cl} 0.15: 0.71, p=0.002)$, how long participants looked at each picture in the first 500ms (500ms epoch dwell: $k=5, g=0.69,95 \% \mathrm{Cl} 0.034: 1.35, p=0.039)$ and how long participants looked at each picture overall (total dwell time: $k=25, g=0.44,95 \% \mathrm{Cl} 0.15: 0.72, p=0.003$ ). Follow-up analyses revealed significant attentional biases on probability of first fixation, latency to first fixation and dwell time for facial stimuli, and number of fixations for sensory word stimuli. Moderator analyses revealed substantial influence of task parameters and some influence of threat status and study quality. Findings support biases in both vigilance and attentional maintenance for pain-related stimuli but suggest attentional biases towards pain are ubiquitous and not related to pain status.
\end{abstract}

Keywords: attentional bias, pain, meta-analysis, eye-tracking, chronic pain, vigilance, avoidance 


\section{Introduction}

It is widely accepted that pain has an interruptive function that demands attention $[3 ; 12 ; 17$;

$42 ; 56 ; 61 ; 69 ; 70]$, however, the impact and experience of pain varies greatly between individuals. Psychological models of chronic pain postulate that the automatic appraisal of pain as highly threatening is a pivotal mechanism that drives pain catastrophising and pain-related fear, which then leads to hypervigilance to pain and pain-related stimuli $[17 ; 56 ; 70]$. This increased vigilance prioritizes potential pain over other information, which activates attempts to either solve the pain [17] or avoid or escape the pain $[69 ; 70]$. The individual's priority to control the pain at all costs interferes with daily life activities and goals [3; 42], which has detrimental effects on mental and physical health, contributing to a vicious cycle of persisting pain and disability [70]. Conversely, individuals who appraise pain as less threatening are proposed to be less vigilant and more likely to remain engaged with valued activities and life goals despite the pain $[17 ; 69 ; 70]$. Considering this, selective attention towards pain and painrelated cues appears to play a fundamental role in the development and maintenance of chronic pain.

Empirical data supports the existence of attentional biases towards pain-related information as measured through experimental paradigms (e.g. dot probe, visual search task) for people with chronic pain, with small but consistent effects across existing meta-analyses [11; 44; 47; 57]. Nevertheless, more recently published effect sizes (e.g.[57]: $d=0.20$ ) are even smaller than earlier meta-analytic estimates (e.g.[47]: $d=0.42$ ), and when attentional biases are detected they appear to be unrelated to theoretically relevant constructs such as pain-related fear or catastrophising [11]. The literature to date might suggest attentional biases towards pain are not as prominent as first proposed. However, paradigms that measure attentional biases have been criticized for having unsatisfactory psychometric properties such as poor consistency, reliability [14] and convergent validity [58] due to their reliance on reaction time indices [40]. 
A clear recommendation for improving the measurement of attentional biases towards pain has been to include eye-tracking methodology during experimental tasks $[11 ; 51 ; 57 ; 63]$ as it provides more direct and reliable measures of where attention is deployed [15; 53]. Not only are eye-tracking measures more reliable, they can more precisely characterize the nature of any attentional processes implicated in chronic pain. Whereas reaction time tasks provide a snapshot of attentional focus for each stimulus exposure, eye-tracking captures the dynamic pattern of attentional processes across the whole task [34; 51]. Additionally, eye-tracking allows assessment of how attention is distributed across multiple simultaneously presented salient stimuli and has led to an increase in more ecologically valid pictorial stimuli, which extends the literature beyond its early focus on word stimuli e.g. $[25 ; 31 ; 32 ; 34 ; 67 ; 68]$.

The aim of this review was to investigate the nature and time course of attentional bias to painrelated stimuli through a meta-analysis of studies using eye-tracking methodology. Specifically, we wanted to determine whether people (children or adults) with chronic pain have greater attentional biases in the initial orienting of attention towards pain-related stimuli (i.e. the vigilance hypothesis) than healthy controls; and/or whether people with chronic pain have greater attentional biases in the subsequent deployment of attention compared to healthy controls (i.e. maintenance and avoidance hypotheses). Our second aim was to determine the role of task parameters and other moderator variables hypothesized to be related to attention to pain including threat status (when manipulated within a study), pain catastrophizing and pain-related fear.

\section{Methods}

\section{Literature search}

This meta-analysis was conducted consistent with the PRISMA guidelines. Relevant published articles were identified through the following electronic databases: MEDLINE, PsychINFO and Web of Science. 
The search strategy was adapted from Crombez et al. [11] and Todd et al. [57] with the addition of terms related to eye-tracking: [selective attention* OR attention*bias* OR vigilance OR hypervigilance OR dot probe OR probe detection OR visual search OR eye adj track* OR eye adj movement* OR gaze] AND pain. Additionally, publication in the English language was set as a search limit. The protocol was registered with PROSPERO on 25 th January 2019, under registration CRD42019119011.

\section{Inclusion criteria}

The following criteria were used to select studies for inclusion in the meta-analysis:

1. The study was a published or in press journal article in English.

2. The study reported data from primary experimental research.

3. The study included participants who reported experiencing chronic pain lasting for 3 months or longer, and/or included participants who did not report a history of chronic pain (i.e. healthy controls). Studies where participants were selected based on a psychiatric disorder rather than a pain disorder were excluded.

4. The study used a behavioural task to measure attentional bias (e.g. visual search, dot-probe, free viewing).

5. The study compared eye movements to pain-related stimuli with eye movements to neutral stimuli on a within-subjects basis.

6. The study assessed attentional bias towards pain-related information, and not towards pain itself (e.g. when visual cues are conditioned to signal pain).

7. The study used eye-tracking technology and reported at least one index of gaze data (i.e. probability of first fixation, latency to first fixation, duration of first fixation, average fixation duration, total dwell time, number of visits). 
8. Data allowing the computation of effect sizes were available. In cases where data were not obtainable from the published research authors were contacted. If authors were unable to provide the data and the effect size could not be calculated, the study was excluded from the meta-analysis (see Figure 1).

The initial search was conducted on 19 March 2019 and resulted in 3260 references, with an updated search performed on 29 October 2019 (EJ), resulting in an additional 200 references (3460 total). All titles and abstracts were screened by one author (EJ) with seven authors (SA, BC, JD, EF, JL, JT, SVD) each independently screening $14.3 \%$ of references (approximately 494 each). There was $100 \%$ consistency between all raters for the inclusion and exclusion of titles and abstracts. Full-text copies of 25 articles were read by two authors (EJ, LS) and 24 were considered eligible for analysis. Figure 1 details the results of the search process. $[19 ; 20 ; 24 ; 25 ; 29-36 ; 41 ; 43 ; 46 ; 48 ; 51 ; 53-55 ; 67 ; 68 ; 72 ; 73]$

Figure 2.1 PRISMA Diagram

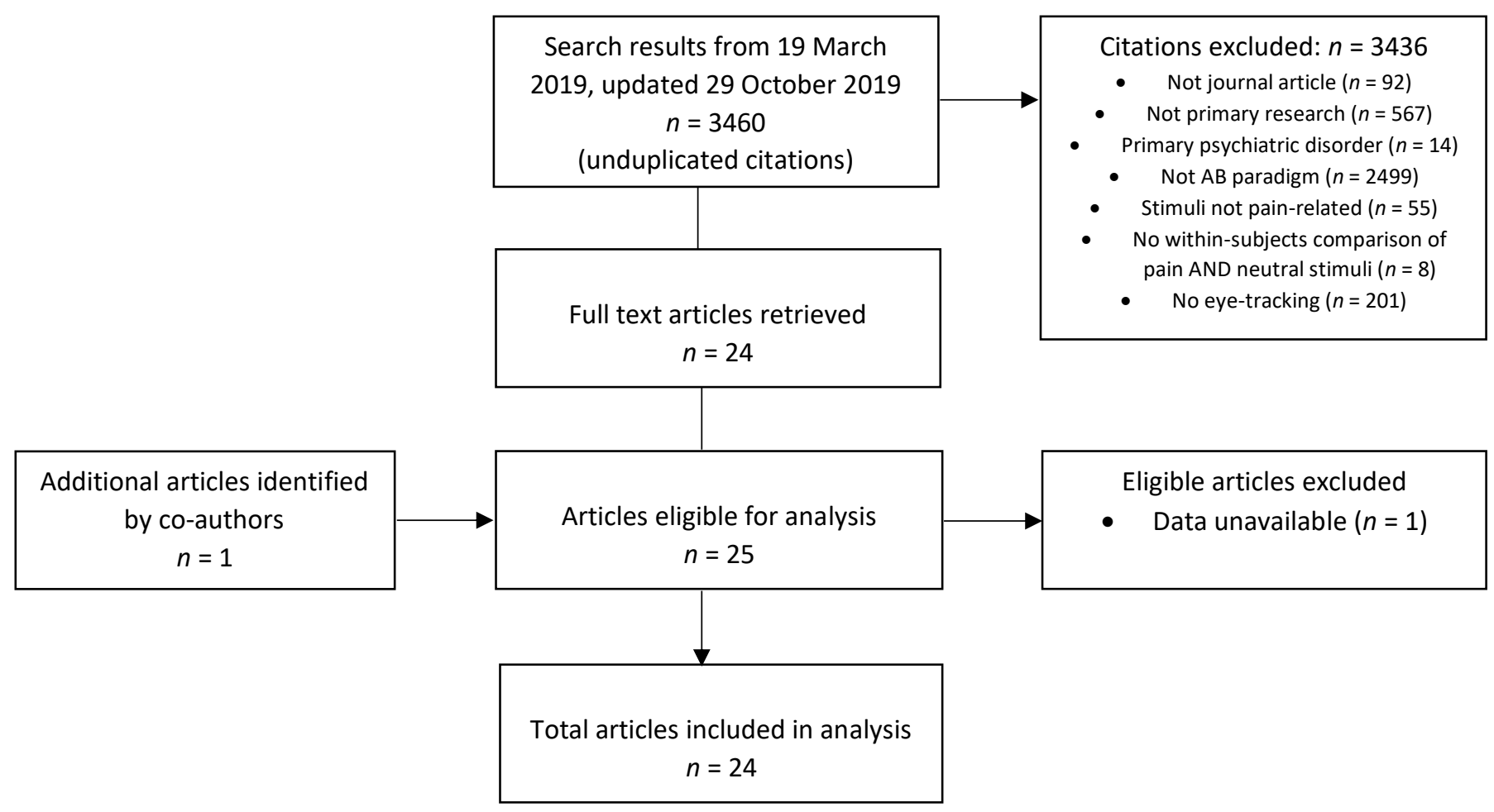




\section{Coding system}

Based on the coding system used by Crombez et al. [11], the team developed and agreed on a protocol for the current meta-analysis outlining the aims, which key variables and moderators would be extracted, the coding scheme for these variables and the criteria for quality ratings.

Coding of study, sample and task parameters was conducted independently (EJ, LS) and then consensus was reached for all discrepancies through discussion. Study characteristics that were coded included year of publication and geographical location. For sample characteristics we coded sample size $(n)$, percentage of males, participants' mean age, SES, whether participants had chronic pain (i.e. pain for more days than not for longer than 3 months [28]; or whether they reported no history of chronic pain and were deemed healthy controls (including those about to undergo an acute experimental pain task). For those samples with chronic pain, we coded for any chronic pain diagnosis, pain intensity/severity, pain location, pain duration and pain-related disability. Previous meta-analyses [4; 11; 57] all highlight the role of task parameters (methodological study characteristics) in moderating attentional bias effect sizes. In line with these prior reviews, we coded for attentional bias paradigm (dot-probe, free viewing, visual search), stimulus modality (faces, pictures, words) and stimulus category. The following stimulus categories were used: pain stimuli (sensory pain words, affective pain words, pain faces, pain-related threat pictures); ill-health stimuli not specifically related to pain (general health threat words); threat stimuli not related to pain or ill-health (general threat words, anger faces, fear faces, sad faces, embarrassed faces); and positive stimuli (happy faces). Also consistent with previous reviews, we coded for stimulus presentation time, proportion of pain trials, proportion of pain stimuli per trial, total number of trials, fixation definition and personal relevance of stimuli.

Similarly, coding of the main outcome data was conducted independently (EJ, LS) and any discrepancies were discussed and resolved. For each eye-tracking variable reported in the manuscript, 
the mean and standard deviation and was recorded. The following eye-tracking variables were coded as early attentional indices indicative of vigilance: probability of first fixation, 500ms epoch dwell (how long participants spent looking at each picture in the first $500 \mathrm{~ms}$ ), latency to first fixation. Duration of first fixation, average fixation duration, total dwell time (how long participants spent overall looking at each picture) and number of fixations were coded as late attentional indices. In terms of theoretically relevant moderators, we coded means and standard deviations for any theoretically relevant variables, including pain catastrophising, fear of pain, threat status (in cases where threat was manipulated) and attentional control.

\section{Risk of Bias}

Risk of bias assessment criteria were adapted from Crombez et al. [11] and were independently double coded (EJ, LS, DVR, TV), with the overall Kappa being substantial $(k=0.77)$. Any ambiguity or disagreements were resolved through discussion until consensus was reached. See supplementary file B for risk of bias criteria.

\section{Protocol Fidelity}

Authors ensured there was high fidelity between the protocol and the review. One variation was a greater number of authors were involved in the title and abstract screening and the quality assessments. The co-authors decided this redistribution of work would be a fairer contribution. Also, due to insufficient data we were unable to analyze type of word stimuli as a task parameter, which was included in the protocol. Finally, in addition to the proposed $\mathrm{I}^{2}$ statistic to measure within-group 
heterogeneity we also included the Q-value, as it has been argued that $\mathrm{I}^{2}$ is insufficient as a measure of heterogeneity [8].

\section{Meta-analytic procedures}

Required datasets for all attentional bias variables were obtained from manuscripts for $60 \%$ of the included studies (EJ, LS). For the remainder of the included studies, first authors provided this data upon request. Given that correlations between the neutral and pain stimuli means were required for analysis but rarely reported in the manuscripts, they were calculated from four available original data sets $[53 ; 55 ; 72 ; 73]$ for all gaze variables. The imputed correlation between the two means were: probability of first fixation ( $r=0.852,95 \% \mathrm{Cl} 0.88$ : 0.99$)$, 500ms epoch dwell $(r=0.495,95 \% \mathrm{Cl} 0.23$ : 0.71), latency to first fixation $(r=0.598,95 \% \mathrm{Cl} 0.47: 0.68)$, duration of first fixation $(r=0.871,95 \% \mathrm{Cl}$ 0.75: 0.85), average fixation duration $(r=0.785,95 \% \mathrm{Cl} 0.60: 0.84)$, total dwell $(r=0.938,95 \% \mathrm{Cl} 0.95$ : 1.03), number of fixations ( $r=0.675,95 \% \mathrm{Cl} 0.59: 0.86)$.

In cases where studies reported multiple results for gaze data variables for the main analyses, the following data types were prioritized in order to maximize power in comparison groups for moderator analyses and to allow for comparison of outcomes with previous reviews [11; 57]: word over pictorial stimuli $[51 ; 54 ; 55]$ and sensory over affective $[51 ; 53 ; 55]$ or health/illness $[54 ; 72 ; 73]$ stimuli. For studies with a between-subjects manipulation (e.g. high vs low threat, attend vs avoid pain) [48; 51; $54 ; 55 ; 68]$, both data sets were included independently, and for studies where data was compared on participant characteristics (e.g. low vs high fear of pain) $[51 ; 72 ; 73]$ an average effect size was calculated using Comprehensive Meta-Analysis [1]. When studies included an impending pain task as a manipulation [29;33], data was taken from the condition without impending pain to ensure comparability with other data sets. 
Comprehensive Meta-Analysis, version 3.3.070 [1] was used to conduct the meta-analytic calculations (EJ). The primary outcome of interest was the standardized paired difference corrected for sample sizes (Hedges' $g$ ) between gaze variables for pain stimuli and neutral stimuli, with a 95\% confidence interval. Hedges' $\mathrm{g}$ is an effect size measure that is appropriate in studies with small samples as it corrects for overestimation of the standard deviation for a population [10]. Hedges and Olkin's [26] guidelines were applied for small $(g=0.1-0.5)$, medium $(g=0.5-0.8)$ and large $(g>0.8)$ effect sizes within and between subjects. For all analyses, a minimum requirement of three studies $(k \geq 3)$ in each comparison group was set. A random effects model was selected for all analyses, which is recommended when including data from multiple independent studies where a common effect size cannot be assumed, therefore assuming heterogeneity [6]. Due to a small number of studies in many of the moderator analyses, a mixed effects model (i.e. a random effects model within subgroups and a fixed effect model across subgroups) was selected. Furthermore, considering the low number of studies in some subgroups, the decision was made to pool within group estimates of Tau². To measure the magnitude of heterogeneity, $\mathrm{I}^{2}$ [27] and Q-value [7] were calculated for each within-group effect size using a critical $p$ value of 0.05 . Heterogeneity between groups was assessed using the $Q$-value significance test [7], also using a critical p of 0.05. Publication bias was examined in primary and followup analyses only in cases where effect sizes were significant. It was assessed using funnel plots, Egger's regression test [18] and Begg and Mazumdar's rank correlation [5]. In addition, Rosenthal's fail-safe N was employed to estimate the number of additional negative studies required to increase the $p$ value above 0.05 [45]. Where results suggested potential publication bias, Duval and Tweedie's trim and fill method [16] was used to calculate an adjusted effect size. Due to multiple variables in the results section, publication bias data is only reported where bias is potentially indicated, however, a full report is included in Supplementary File A. 
The analyses were structured in four stages. First, within-group comparisons were conducted individually for the chronic pain and control groups across all 7 attentional bias gaze variables. Next, between-group analyses were performed across studies that included both chronic pain and control participants. Then, separate subgroup analyses were conducted for attentional biases towards sensory words, undifferentiated pain pictures, and pain faces to explore the effect of stimulus type. Finally, we conducted moderator analyses to investigate the role of theoretically relevant variables (e.g. threat status, pain catastrophising) and the impact of task parameters on gaze variables where heterogeneity was significant $(p<0.05)$ and where each group included at least three studies e.g.[57].

\section{Results}

\section{Descriptive statistics}

Table 1 provides a summary of included studies. There was a total of 24 studies containing 23 unique samples that comprised a total sample of $N=1,425$. The average sample size for the chronic pain groups $(\mathrm{k}=9)$ was $36(\mathrm{SD}=23, \mathrm{~N}=326)$ and for the control groups $(\mathrm{k}=21)$ was $52(\mathrm{SD}=31, \mathrm{~N}=$ 1,099). Of these studies, 23 included an adult sample whereas one study included a child sample [25]. There were 11 studies that used the dot-probe task, comprising 14 independent groups ( 9 control groups, 5 pain groups), with one of these studies [29] also using a visual cueing task with the same control sample. There were 11 studies that used a free-viewing task, comprising 13 independent groups (10 control groups, 3 pain groups). One study used a visual search task [46] comprising 2 independent groups (1 control group, 1 pain group) and one study [33] used a perceptual task manipulation (impending touch vs pain) whilst measuring attentional biases to images ( 1 control group). Of the 24 studies, 12 were within-subject designs and 12 were within and between-subject designs. 
Table 1. Summary of Included Studies

\begin{tabular}{|c|c|c|c|c|c|c|c|c|c|c|}
\hline \multirow[b]{2}{*}{$\begin{array}{l}\text { Study } \\
\text { (Author, } \\
\text { Year) }\end{array}$} & \multirow[b]{2}{*}{$\begin{array}{l}\text { Sample type } \\
\text { and size (n) }\end{array}$} & \multirow[b]{2}{*}{ Paradigm } & \multirow[b]{2}{*}{ Stimuli } & \multicolumn{7}{|c|}{ Eye-tracking measures (Hedge's $g, S E$ ) } \\
\hline & & & & $\begin{array}{l}\text { Probability } \\
\text { FF }\end{array}$ & $\begin{array}{l}\text { 500ms } \\
\text { epoch } \\
\text { dwell }\end{array}$ & Latency FF & Duration FF & $\begin{array}{l}\text { Average } \\
\text { fixation } \\
\text { duration }\end{array}$ & Total dwell & $\begin{array}{l}\text { Number } \\
\text { fixations }\end{array}$ \\
\hline $\begin{array}{l}\text { Fashler et } \\
\text { al., } 2014\end{array}$ & $\begin{array}{l}\text { Control }(n=62) \\
\text { Pain }(n=51)\end{array}$ & Dot-probe & Words & - & $\begin{array}{l}0.13(0.13) \\
0.19(0.14)\end{array}$ & - & - & $\begin{array}{l}0.002(0.08) \\
0.007(0.09)\end{array}$ & $\begin{array}{l}0.17(0.05) \\
0.40(0.05)\end{array}$ & $\begin{array}{l}0.26(0.10) \\
0.59(0.12)\end{array}$ \\
\hline $\begin{array}{l}\text { Fashler et } \\
\text { al., } 2016\end{array}$ & $\begin{array}{l}\text { Control }(n=62) \\
\text { Pain }(n=51)\end{array}$ & Dot-probe & Pictures & - & $\begin{array}{l}0.33(0.13) \\
0.03(0.14)\end{array}$ & - & - & $\begin{array}{l}-0.03(0.13) \\
0.01(0.14)\end{array}$ & $\begin{array}{l}-0.06(0.13) \\
0.45(0.15)\end{array}$ & $\begin{array}{l}1.27(0.17) \\
0.59(0.15)\end{array}$ \\
\hline $\begin{array}{l}\text { Franklin et } \\
\text { al., } 2019\end{array}$ & $\begin{array}{l}\text { Control }(n=17) \\
\text { Pain }(n=18)\end{array}$ & Dot-probe & Pictures & - & - & - & $\begin{array}{l}0.30(0.12) \\
-0.46(0.12)\end{array}$ & $\begin{array}{l}-0.49(0.16) \\
0.65(0.16)\end{array}$ & - & - \\
\hline $\begin{array}{l}\text { Heathcote } \\
\text { et al., } 2017\end{array}$ & Control $(n=37)$ & Free-viewing & Faces & $0.39(0.09)$ & - & - & - & - & $0.57(0.06)$ & - \\
\hline $\begin{array}{l}\text { Jackson et } \\
\text { al., } 2018\end{array}$ & Control $(n=82)$ & Dot-probe & Pictures & $1.42(0.08)$ & - & $0.13(0.09)$ & $0.038(0.05)$ & - & $0.68(0.04)$ & $0.62(0.10)$ \\
\hline $\begin{array}{l}\text { Jackson et } \\
\text { al., } 2019\end{array}$ & Pain $(n=89)$ & Dot-probe & Pictures & $1.98(0.09)$ & - & - & - & - & $1.76(0.06)$ & - \\
\hline $\begin{array}{l}\text { Kasewater } \\
\text { et al., } 2019\end{array}$ & Control $(n=138)$ & Free-viewing & Faces & - & - & $-1.06(0.09)$ & - & - & $1.85(0.05)$ & - \\
\hline $\begin{array}{l}\text { Lee et al., } \\
2018\end{array}$ & Pain $(n=50)$ & Free-viewing & Faces & - & $-0.26(0.17)$ & - & - & - & $0.58(0.05)$ & - \\
\hline $\begin{array}{l}\text { Liossi et al., } \\
2014\end{array}$ & $\begin{array}{l}\text { Control }(n=23) \\
\text { Pain }(n=23)\end{array}$ & Free-viewing & Faces & $\begin{array}{l}0.07(0.11) \\
0.77(0.13)\end{array}$ & - & - & $\begin{array}{l}0.12(0.10) \\
-0.18(0.10)\end{array}$ & $\begin{array}{l}0.008(0.13) \\
-0.20(0.13)\end{array}$ & - & $\begin{array}{l}-0.26(0.17) \\
-0.42(0.17)\end{array}$ \\
\hline $\begin{array}{l}\text { Ling et al., } \\
2019\end{array}$ & Control $(n=64)$ & $\begin{array}{l}\text { Somatosenso } \\
\text { ry detection } \\
\text { task }\end{array}$ & Faces & $0.85(0.08)$ & - & - & $0.44(0.06)$ & - & $0.59(0.05)$ & - \\
\hline $\begin{array}{l}\text { Mahmoodi } \\
\text { et al., } 2019\end{array}$ & $\begin{array}{l}\text { Control }(n=18) \\
\text { Pain }(n=20)\end{array}$ & Free-viewing & Pictures & $\begin{array}{l}1.16(0.24) \\
1.20(0.16)\end{array}$ & - & $\begin{array}{l}0.34(0.21) \\
-0.14(0.19)\end{array}$ & $\begin{array}{l}-0.24(0.15) \\
0.17(0.11)\end{array}$ & $\begin{array}{l}0.24(0.15) \\
-0.13(0.14)\end{array}$ & - & $\begin{array}{l}1.17(0.24) \\
1.79(0.29)\end{array}$ \\
\hline
\end{tabular}




\begin{tabular}{|c|c|c|c|c|c|c|c|c|c|c|}
\hline $\begin{array}{l}\text { Mazidi et al., } \\
2019\end{array}$ & $\begin{array}{l}\text { Control }(n=29) \\
\text { Pain }(n=28)\end{array}$ & Dot-probe & Faces & $\begin{array}{l}0.0(0.09) \\
0.43(0.10)\end{array}$ & - & $\begin{array}{l}-0.08(0.16) \\
0.31(0.17)\end{array}$ & $\begin{array}{l}-0.004(0.09) \\
0.11(0.09)\end{array}$ & - & $\begin{array}{l}-0.28(0.07) \\
-0.14(0.07)\end{array}$ & $\begin{array}{l}-0.22(0.15) \\
-0.26(0.15)\end{array}$ \\
\hline $\begin{array}{l}\text { Peters et al., } \\
2016\end{array}$ & Control $(n=56)$ & Free-viewing & Faces & - & - & - & - & - & $0.10(0.05)$ & - \\
\hline $\begin{array}{l}\text { Priebe et al., } \\
2015\end{array}$ & Control $(n=48)$ & Free-viewing & Faces & $-0.66(0.09)$ & $4.16(0.44)$ & - & - & $0.86(0.11)$ & $4.48(0.17)$ & - \\
\hline $\begin{array}{l}\text { Schoth et } \\
\text { al., } 2015\end{array}$ & $\begin{array}{l}\text { Control }(n=24) \\
\text { Pain }(n=23)\end{array}$ & $\begin{array}{l}\text { Visual Search } \\
\text { Task }\end{array}$ & Faces & $\begin{array}{l}0.74(0.12) \\
0.83(0.13)\end{array}$ & - & $\begin{array}{l}-0.99(0.22) \\
-1.17(0.24)\end{array}$ & - & - & - & - \\
\hline $\begin{array}{l}\text { Schoth et } \\
\text { al., } 2019\end{array}$ & Control $(n=55)$ & Free-viewing & Pictures & - & - & $0.56(0.15)$ & - & $0.34(0.09)$ & - & $-0.36(0.11)$ \\
\hline $\begin{array}{l}\text { Sharpe et } \\
\text { al., } 2017\end{array}$ & Control $(n=98)$ & Dot-probe & $\begin{array}{l}\text { Words } \\
\text { Faces }\end{array}$ & $\begin{array}{l}-0.03(0.10) \\
0.22(0.05)\end{array}$ & - & $\begin{array}{l}-0.12(0.09) \\
0.099(0.08)\end{array}$ & $\begin{array}{l}-0.14(0.05) \\
0.19(0.05)\end{array}$ & - & $\begin{array}{l}-0.09(0.04) \\
0.091 \\
(0.03)\end{array}$ & - \\
\hline $\begin{array}{l}\text { Skinner et } \\
\text { al., } 2018\end{array}$ & Control $(n=49)$ & Free-viewing & Words & $-0.25(0.08)$ & - & $0.04(0.13)$ & $-0.15(0.07)$ & - & $-0.27(0.05)$ & $-0.04(0.11)$ \\
\hline $\begin{array}{l}\text { Sun et al., } \\
2016\end{array}$ & Control $(n=60)$ & Dot-probe & $\begin{array}{l}\text { Words } \\
\text { Pictures }\end{array}$ & - & - & $\begin{array}{l}-0.06(0.13) \\
-0.11(0.16)\end{array}$ & $\begin{array}{l}0.20(0.12) \\
0.17(0.15)\end{array}$ & - & $\begin{array}{l}0.21(0.18) \\
0.55(0.16)\end{array}$ & - \\
\hline $\begin{array}{l}\text { Todd et al., } \\
2016\end{array}$ & Control $(n=86)$ & Dot-probe & $\begin{array}{l}\text { Words } \\
\text { Faces }\end{array}$ & $\begin{array}{l}0.23(0.09) \\
0.11(0.06)\end{array}$ & - & $\begin{array}{l}-0.08(0.09) \\
-0.02(0.15)\end{array}$ & $\begin{array}{l}-0.10(0.06) \\
0.15(0.10)\end{array}$ & - & $\begin{array}{l}-0.05(0.04) \\
0.10(0.12)\end{array}$ & - \\
\hline $\begin{array}{l}\text { Vervoort et } \\
\text { al., } 2013\end{array}$ & Control $(n=35)$ & Free-viewing & Faces & - & - & $-6.51(0.71)$ & $-2.07(0.15)$ & - & $-0.47(0.06)$ & $-0.38(0.14)$ \\
\hline $\begin{array}{l}\text { Vervoort et } \\
\text { al., } 2014\end{array}$ & Control $(n=62)$ & Free-viewing & Faces & - & - & $0.03(1.1)$ & - & - & $0.01(2.23)$ & $-2.24(0.22)$ \\
\hline $\begin{array}{l}\text { Yang et al., } \\
2012\end{array}$ & Control $(n=41)$ & Dot-probe & Words & $0.31(0.09)$ & - & $0.11(0.14)$ & $0.29(0.08)$ & $0.02(0.01)$ & $0.54(0.06)$ & $0.38(0.13)$ \\
\hline $\begin{array}{l}\text { Yang et al., } \\
2013\end{array}$ & $\begin{array}{l}\text { Control }(n=24) \\
\text { Pain }(n=24)\end{array}$ & Dot-probe & Words & $\begin{array}{l}0.25(0.11) \\
-0.63(0.12)\end{array}$ & - & - & $\begin{array}{l}-0.16(0.10) \\
0.07(0.1)\end{array}$ & $\begin{array}{l}-0.08(0.13) \\
-0.11(0.13)\end{array}$ & $\begin{array}{l}0.43(0.07) \\
-0.13(0.07)\end{array}$ & $\begin{array}{l}0.30(0.16) \\
0.04(0.16)\end{array}$ \\
\hline
\end{tabular}

Note: Fashler (2014) and Fashler (2016) used the same sample. 


\section{Risk of Bias}

Comprehensive quality analysis information can be found in Supplementary File B. Three quarters of the available studies (79\%) fulfilled all external validity criteria, and more than half (58.3\%) fulfilled the internal validity criteria. All individual items fulfilled criteria in more than $60 \%$ of studies, except for participant engagement with task (33\% of studies), which accounts for the comparably poorer internal validity reported above. The risk of bias in this meta-analysis is comparable to the risk of bias in the two most recent meta-analyses [11; 57]. Although both meta-analyses had different inclusion criteria, their findings and conclusions were consistent and thus likely reflective of the actual effects in those studies. Therefore, the authors estimate that we can be moderately confident in the estimates of effect sizes observed in this meta-analysis.

\section{Do people with and/or without chronic pain show a bias towards pain-related compared to neutral stimuli in indices of early attention?}

The within-groups effect sizes $(\mathrm{g})$, 95\% confidence intervals ( $95 \% \mathrm{Cl}$ ), number of independent groups (k), and number of participants ( $\mathrm{n}$ ) for the chronic pain and healthy control groups for all gaze variables are reported in Table 2. A sensitivity analysis was conducted on relevant main analyses with and without the only child study, and the pattern of results remained unchanged. Therefore, we report the full analyses below.

\section{Probability of first fixation}

Overall, the effect size for probability of first fixation indicated a small and significant attentional bias $(\mathrm{k}=21, g=0.43,95 \% \mathrm{Cl} 0.15: 0.71, \mathrm{p}=0.002)$ That is, people were more likely to look at the pain word or picture first compared the non-pain stimuli, but there was substantial heterogeneity $\left(\mathrm{Q}_{19}=\right.$ 
813.53, $\left.\mathrm{I}^{2}=97.84, \mathrm{p}<.001\right)$. A medium sized effect was found for the chronic pain group $(k=6, g=0.76$, $95 \% \mathrm{Cl} 0.014: 1.51, \mathrm{p}=0.003)$, and small but significant effect was observed for the control group $(\mathrm{k}=$ $15, g=0.30,95 \% \mathrm{Cl} 0.03: 0.58, p=0.049)$. The between group difference for probability of first fixation between pain and control participants was not significant $\left(Q_{1}=1.29, p=0.13\right)$. Further, the direct between-groups analysis revealed no significant group difference $(k=5, g=0.68,95 \% \mathrm{Cl}-0.07: 1.44, p$ $=0.08$ ). For all studies combined there was asymmetry evident in the Funnel plot upon visual inspection, and Begg and Mazumdar's rank correlation was significant for this combined effect (Tau $=0.31, p=$ 0.049). However, Egger's regression was not significant $(t=1.42, p=0.17)$ and the fail-safe $n$ was large ( $n=1781$ ), indicating little evidence of publication bias. Further, when Duval and Tweedies' trim and fill was applied, no studies were trimmed. Hence, both the chronic pain and control groups demonstrated a bias to pain relative to non-pain stimuli.

\section{0ms Epoch Dwell}

The combined effect for 500ms epoch dwell was moderate and significant $(k=5, g=0.69,95 \%$ $\mathrm{Cl} 0.03: 01.35, p=0.04)$, but with substantial heterogeneity $\left(Q_{3}=83.07, I^{2}=95.53, p<.001\right)$. This indicates participants spent longer over the first $500 \mathrm{~ms}$ looking at the pain stimuli relative to non-pain stimuli. There was an insufficient number of studies to analyse the effect size for $500 \mathrm{~ms}$ epoch dwell for chronic pain participants $(k=2)$, however, for the control group the same was found and the effect was large $(\mathrm{k}=3, g=1.37,95 \% \mathrm{Cl} 0.08: 2.67, \mathrm{p}=0.01)$, with substantial heterogeneity $\left(\mathrm{I}^{2}=77.58, \mathrm{p}<.001\right)$. The difference between groups was not significant $\left(Q_{1}=2.83, p=0.09\right)$. For all studies combined there was asymmetry evident in the Funnel plot upon visual inspection. Egger's regression was significant $(t=4.52$, $p=0.02)$ and the fail-safe $n$ was relatively low $(n=25)$. However, Begg and Mazumdar's rank correlation was not significant (Tau $=0.10, p=0.81$ ), indicating some evidence of publication bias. No studies were 
trimmed following Duval and Tweedie's trim and fill adjustment, as such the overall effect remained unchanged.

\section{Latency to first fixation}

The combined effect size was not significant $(\mathrm{k}=21, g=0.2395 \% \mathrm{Cl}-0.51: 0.04, \mathrm{p}=0.01)$, however, there was substantial heterogeneity $\left(Q_{19}=324.11, I^{2}=93.83, p<.001\right)$. The effect size for latency to first fixation was not significant for the chronic pain group $(\mathrm{k}=3, g=-0.33,95 \% \mathrm{Cl}-1.12: 0.49$, $\mathrm{p}=0.40)$, nor the control group $(\mathrm{k}=18, g=-0.22,95 \% \mathrm{Cl}-0.51: 0.08, \mathrm{p}=0.15)$ and the difference between groups was not significant $\left(Q_{1}=0.05, p=0.81\right)$. That is, there was no difference in the time taken for participants to fixate initially on pain vs non-pain stimuli. Consistent with this, the direct between-groups analysis was also not significant $(k=3, g=0.31,95 \% \mathrm{Cl}-0.08: 0.69, p=0.12)$.

\section{Summary}

In terms of indices of early attention, people with and without pain were significantly more likely to look at the pain stimuli first on each trial compared with non-pain stimuli and spent longer in the first $500 \mathrm{~ms}$ looking at pain stimuli relative to non-pain stimuli. There was no significant difference for either pain or control participants when exploring the time taken to initially fixate on pain vs nonpain stimuli, and there were no significant differences found on any of these variables between people with chronic pain and control groups.

\section{Do people with and/or without chronic pain show a bias towards pain-related compared to neutral stimuli in indices of sustained attention?}

\section{Duration of First Fixation}


The overall effect size for duration of first fixation was not significant $(k=21, g=-0.07,95 \% \mathrm{Cl}$ 0.21: $0.07, p=0.32)$ but there was substantial heterogeneity $\left(Q_{20}=309.37, I^{2}=93.54, p<.001\right)$. There was no significant effect on duration of first fixation for the chronic pain group $(\mathrm{k}=5, g=-0.05,95 \% \mathrm{Cl}-$ 0.26: $0.16, p=0.63)$ nor the control group $(k=16, g=-0.08,95 \% \mathrm{Cl}-0.26: 0.10, p=0.37)$. The between group difference was also not significant $\left(Q_{1}=0.44, p=0.83\right)$. This indicates no difference for either group on the time spent looking at the pain or non-pain stimuli on the first fixation. Further, there was no evidence of direct between-group differences $(k=5, g=-0.45,95 \% \mathrm{Cl}-1.09: 0.20, p=0.18)$.

\section{Average Fixation Duration}

Similarly, the overall combined effect size for average fixation duration was not significant $(k=$ $14, g=0.09,95 \% \mathrm{Cl}-0.08: 0.26, p=0.29)$. However, there was evidence of heterogeneity $\left(Q_{12}=91.50, I^{2}\right.$ $=86.58, \mathrm{p}<.001)$. Consistent with the combined data, there was no effect for average fixation duration for either the chronic pain $(k=5, g=0.03,95 \% \mathrm{Cl}-0.22: 0.28, \mathrm{p}=0.80)$ or control group $(\mathrm{k}=9, g=0.09$, $95 \% \mathrm{Cl}-0.09: 0.38, p=0.23)$, nor was there a difference between groups $\left(Q_{1}=0.42, p=0.51\right)$. That is, no difference was detected between the average fixation lengths of pain and non-pain stimuli. Further, there was no evidence of direct between-group differences $(k=5, g=0.21,95 \% \mathrm{Cl}-0.37: 0.79, p=0.48)$.

\section{Total Dwell Time}

The combined effect size for total dwell time indicated a small and significant attentional bias (k $=25, g=0.44,95 \% \mathrm{Cl} 0.15: 0.72, p=0.003)$, but again there was significant heterogeneity $\left(Q_{23}=3704, I^{2}\right.$ $=99.37, \mathrm{p}<.001)$. That is, participants spent longer overall looking at the pain stimuli compared to nonpain stimuli. The effect for the chronic pain group was not significant $(\mathrm{k}=5, g=0.50,95 \% \mathrm{Cl}-0.15: 1.14$, $p=0.13)$ but there was a small and significant effect for the control group in the same direction $(k=20$, $g=0.42,95 \% \mathrm{Cl} 0.10: 0.74, \mathrm{p}=.010)$. The between group difference was not, however, significant $\left(\mathrm{Q}_{1}=\right.$ 
$0.044, p=0.83)$. Direct between-group analyses also revealed no significant differences between chronic pain and control participants $(k=3, g=0.10,95 \% \mathrm{Cl}-0.77: 0.96, p=0.83)$.

\section{Number of Fixations}

For number of fixations, the overall effect was not significant $(\mathrm{k}=18, g=-0.08,95 \% \mathrm{Cl}-0.38$ :

$0.21, p=0.59)$, however, there was evidence of heterogeneity $\left(Q_{16}=305.31, I^{2}=94.51, p=<.001\right)$. There was no evidence of attentional bias for either group: chronic pain $(\mathrm{k}=5, g=0.31,95 \% \mathrm{Cl}-0.27: 0.89, \mathrm{p}=$ 0.29 ) or control groups $(\mathrm{k}=13, g=-0.23,95 \% \mathrm{Cl}-0.59: 0.12, \mathrm{p}=0.20)$. The between group difference was also not significant $\left(Q_{1}=2.49, p=0.11\right)$. This indicates there was no difference for either group on the number of fixations to pain vs no-pain stimuli. Consistent with this, there was no evidence of direct between-group differences $(k=5, g=0.08,95 \% \mathrm{Cl}-0.15: 0.30, p=0.50)$.

\section{Summary}

In summary, for indices of later attention, a significant difference was found for the combined chronic pain and control sample and the control group alone on total dwell time, such that participants spent longer overall looking at pain stimuli relative to non-pain stimuli. No significant differences were found for either group on duration of first fixation, average fixation duration, or number of fixations, and there was no evidence of differences between chronic pain participants and control participants on any of the late attentional indices. 
Table 2. Primary Analyses

\begin{tabular}{|c|c|c|c|c|c|c|}
\hline Variable & $k$ & $n$ & Hedges'g & $95 \% \mathrm{Cl}$ & $\mathrm{I}^{2}$ & $\mathrm{Q}_{\mathrm{B}}$ \\
\hline Probability First Fixation & 21 & 839 & $0.43^{* *}$ & 0.15 to 0.71 & $97.84 * * *$ & 1.29 \\
\hline Chronic Pain & 6 & 207 & $0.76^{*}$ & 0.01 to 1.51 & $98.38 * * *$ & \\
\hline Controls & 15 & 632 & $0.30 *$ & 0.03 to 0.58 & $97.25 * * *$ & \\
\hline 500ms Epoch Dwell & 5 & 260 & $0.69 *$ & 0.03 to 1.35 & $95.53 * * *$ & 2.83 \\
\hline Controls & 3 & 159 & $1.37^{*}$ & 0.08 to 2.67 & $77.58 * * *$ & \\
\hline Latency to First Fixation & 21 & 839 & -0.23 & -0.51 to 0.04 & $93.83 * * *$ & 0.053 \\
\hline Chronic Pain & 3 & 71 & -0.33 & -1.12 to 0.49 & $92.24 * * *$ & \\
\hline Controls & 18 & 768 & -0.22 & -0.51 to 0.08 & $94.29 * * *$ & \\
\hline Duration First Fixation & 21 & 732 & -0.08 & -0.23 to 0.08 & $93.54 * * *$ & 0.44 \\
\hline Chronic Pain & 5 & 113 & -0.05 & -0.26 to 0.16 & $81.01 * * *$ & \\
\hline Controls & 16 & 619 & -0.08 & -0.26 to 0.10 & $94.80 * * *$ & \\
\hline Average Fixation Duration & 14 & 424 & 0.09 & -0.08 to 0.26 & $86.58^{* * *}$ & 0.42 \\
\hline Chronic Pain & 5 & 136 & 0.03 & -0.22 to 0.28 & $79.40 * * *$ & \\
\hline Controls & 9 & 288 & 0.09 & -0.09 to 0.38 & $88.90 * * *$ & \\
\hline Total Dwell & 25 & 1204 & $0.44 * *$ & 0.15 to 0.72 & $99.37 * * *$ & 0.044 \\
\hline Chronic Pain & 5 & 242 & 0.50 & -0.15 to 1.14 & $99.37^{* * *}$ & \\
\hline Controls & 20 & 962 & $0.42 * *$ & 0.10 to 0.74 & $99.39 * * *$ & \\
\hline Number of Fixations & 18 & 624 & -0.08 & -0.38 to 0.21 & $94.51 * * *$ & 2.49 \\
\hline Chronic Pain & 5 & 146 & 0.31 & -0.27 to 0.89 & $93.82 * * *$ & \\
\hline Controls & 13 & 478 & -0.23 & -0.59 to 0.12 & $95.01 * * *$ & \\
\hline
\end{tabular}

Note. $k$ values are the number of effect sizes; Hedges' $g$ values are the standardized differences of attentional biases between pain and neutral stimuli (small effect $=0.1-0.5$, medium effect $=0.5-0.8$, and large effect $>0.8$ ); $\mathrm{Cl}=$ confidence interval; $I^{2}$ values represent the within group heterogeneity statistic; $\mathrm{Q}_{\mathrm{B}}$ values represent the between group difference statistic.

$* p<.05 ; * * p<.01 ; * * * p<.001$ 


\section{Are attentional biases stimulus-specific (sensory pain words, pain pictures and pain faces)?}

Consistent with previous meta-analyses [11; 57], we wanted to explore the stimulus specificity of attentional biases by calculating effect sizes for pain words and pain pictures separately. Due to limited studies including word stimuli, only sensory words were investigated. Similarly, in the interest of consistency with previous meta-analyses, we analyzed pain pictures as an undifferentiated group (i.e. painful facial expressions and injury-related pictures). However, we also ran analyses with pain faces only, as there has been an increase of eye-tracking studies using face stimuli in the literature. Considering there were no significant direct between-group differences on any of the gaze variables, chronic pain and control participants were combined in all subsequent analyses. However, where there were sufficient studies, we also investigated chronic pain vs healthy participant group as a moderator of the effect. Significant results are reported below, full analyses can be found in Supplementary File C.

\section{Sensory Pain Words}

Attentional bias towards sensory pain words was only significant for number of fixations $(k=6, g$ $=0.26,95 \% \mathrm{Cl} 0.07: 0.45, \mathrm{p}=0.008)$, indicating that people had more fixations on sensory pain words than neutral words. There were insufficient studies in the chronic pain group $(k=2)$ to compare this effect with healthy control groups.

\section{$\underline{\text { Pain Pictures }}$}

In contrast to findings for pain-related words, overall there was a significant moderate effect for pain pictures on probability of first fixation $(k=13, \mathrm{~g}=0.70,95 \% \mathrm{Cl} 0.30: 1.11, \mathrm{p}=0.001)$. That is, people were more likely to look at the pain picture first before neutral picture/s. When analyzed separately this effect was numerically larger for the chronic pain participants $(\mathrm{k}=5, g=1.04,95 \% \mathrm{Cl} 0.42: 1.67, \mathrm{p}=$ $0.001)$ than the control group $(\mathrm{k}=8, g=0.49,95 \% \mathrm{Cl}-0.001: 0.99, \mathrm{p}=0.05)$, however, the difference 
between the two groups did not reach significance $\left(Q_{1}=1.82, p=0.18\right)$. There was also a significant moderate effect overall for dwell time $(\mathrm{k}=13, g=0.74,95 \% \mathrm{Cl} 0.22: 1.26, \mathrm{p}=0.005)$, indicating people spent longer overall looking at pain compared to non-pain pictures. Although, when analyzed separately this was only significant for controls $(\mathrm{k}=10, g=0.75,95 \% \mathrm{Cl} 0.12: 1.37, \mathrm{p}=0.02)$ and not for pain participants $(\mathrm{k}=3, g=0.74,95 \% \mathrm{Cl}-0.40: 1.78, \mathrm{p}=0.20)$. However, this difference was not significant $\left(Q_{1}=0.00, p=0.99\right)$.

\section{$\underline{\text { Pain Faces }}$}

A small, significant overall effect was also found towards pain faces compared to neutral faces on probability of first fixation ( $k=12, g=0.32,95 \% \mathrm{Cl} 0.08$ : $0.56, p=0.01$ ), showing people were more likely to look at the pain face first. However, individual analyses revealed this effect was only significant for chronic pain participants $(k=3, g=0.67,95 \% \mathrm{Cl} 0.41: 0.92, \mathrm{p}<.001)$ and not controls $(\mathrm{k}=9, g=0.20$, $95 \% \mathrm{Cl}-0.07: 0.48, p=0.15)$ and this difference was significant $\left(Q_{1}=5.94, p=0.02\right)$. A moderate significant effect was found for latency to first fixation overall $(\mathrm{k}=11, g=-0.62,95 \% \mathrm{Cl}-1.09:-0.14, \mathrm{p}=$ 0.01), indicating people were quicker to fixate initially on pain faces compared to neutral faces. There were insufficient studies in the chronic pain group $(k<3)$ to compare individual group analyses. Similarly, for dwell time, a moderate significant effect was found indicating all participants spent longer looking at painful facial expressions over neutral expressions $(\mathrm{k}=14, g=0.54,95 \% \mathrm{Cl} 0.10: 0.97, \mathrm{p}=$ 0.02). In contrast, for number of fixations a large significant effect was also observed, but in the opposite direction indicating participants made fewer individual fixations on painful facial expressions compared to neutral $(k=7, g=-0.82,95 \% \mathrm{Cl}-1.33:-0.32, p=0.001)$. In both cases, analyses between groups could not be performed due to $\mathrm{k}<3$ studies in the chronic pain group. Asymmetry was evident in the Funnel plot upon visual inspection and Egger's regression co-efficient was significant for number of fixations $(t=7.09, p<.001)$ as was Begg and Mazumdar's rank correlation (Tau $=-0.67, p=0.04)$. Even 
though the fail-safe $n$ was relatively large $(n=175)$, evidence of publication bias seems likely for this outcome. However, Duval and Tweedie's trim-and-fill method resulted in no studies being trimmed, and therefore the effect remained the same.

\section{Summary}

In summary, participants had more fixations on sensory pain words compared to neutral words but were more likely to look at pain pictures first compared to neutral and spent longer overall looking at pain compared to neutral pictures. For facial stimuli, people with chronic pain were more likely than those without pain to look at the painful facial expression first relative to the neutral facial expression. All participants were quicker to look at and spent longer overall looking at the pain rather than neutral face, however, they fixated fewer times on the pain face compared to the neutral face.

\section{Do relevant theoretical variables moderate attentional biases?}

We planned to investigate threat status as a categorical moderator, and pain catastrophising, fear of pain and attentional control as continuous moderators. Due to an insufficient number of studies in comparison groups $(\mathrm{k}<3)$, the relationship between threat status and average fixation was not analyzed. In terms of continuous moderators, there was insufficient data to analyze attentional control and to examine the effect of catastrophising and fear of pain on average fixation duration and number of fixations.

\section{$\underline{\text { Threat Status }}$}

Under low threat manipulations, the overall effect was significant for latency to first fixation $(k=$ $15, g=-0.35,95 \% \mathrm{Cl}-0.67:-0.01, p=0.04)$ and dwell time $(\mathrm{k}=19, g=0.53,95 \% \mathrm{Cl} 0.20: 0.86, p=0.001)$, but these were not significant under high threat manipulations (latency to first fixation: $k=6, g=0.03$, 
$95 \% \mathrm{Cl}-0.44:-0.50, p=0.91 ; \mathrm{dwell}: \mathrm{k}=6, g=0.14,95 \% \mathrm{Cl}-0.54: 0.83, p=0.69)$. That is, participants under low threat conditions responded more quickly to pain-related than neutral stimuli on the first fixation and maintained gaze on pain-related stimuli longer than neutral stimuli. However, the difference between threat groups was not significant (latency to first fixation: $Q_{1}=1.64, p=0.20 ; d$ well: $\left.Q_{1}=1.00, p=0.32\right)$. In contrast, for number of fixations the observed effect was significant for high threat $(\mathrm{k}=3, g=-1.60,95 \% \mathrm{Cl}-3.05:-0.15, p=0.03)$ but not low threat groups $(\mathrm{k}=15, g=0.18,95 \% \mathrm{Cl}$ 0.06: $0.42, p=0.14)$, and this difference between threat groups was significant $\left(Q_{1}=5.65, p=0.02\right)$. This finding indicates that under conditions of high threat, fewer fixations were made to pain-related stimuli than neutral stimuli. For probability of first fixation, the effect was small and significant for both high ( $\mathrm{k}$ $=3, g=0.25,95 \% \mathrm{Cl} 0.02: 0.47, p=0.03)$ and low $(\mathrm{k}=18, g=0.46,95 \% \mathrm{Cl} 0.13: 0.80, p=0.006)$ groups, with no significant difference between the two $\left(Q_{1}=1.14, p=0.29\right)$.

\section{$\underline{\text { Pain Catastrophizing and Fear of Pain }}$}

Pain catastrophising accounted for a very small but significant amount of variance for probability of first fixation $(\mathrm{k}=7, r=0.03,95 \% \mathrm{Cl} 0.002: 0.05, \mathrm{p}=0.03)$, indicating that people were more likely to look at the pain stimulus compared to non-pain stimulus first if they had higher levels of reported pain catastrophising. However, pain catastrophising was not significantly associated with any other gaze variables. Fear of pain did not account for a significant amount of variance for any attentional bias variables.

\section{Do task parameters moderate attentional biases?}

We planned to investigate the following categorical moderators: paradigm (dot probe vs freeviewing), stimulus modality (words vs pictures), fixation definition ( $\geq 60 \mathrm{~ms}$ vs $\geq 100 \mathrm{~ms}$ ), stimuli 
presentation time ( $\leq 2000 \mathrm{~ms}$ vs $>2000 \mathrm{~ms}$ ), proportion of pain trials ( $\leq 50 \%$ vs $>50 \%$ ), proportion of pain stimuli per trial and personal relevance of pain stimuli. Due to insufficient studies $(k<3)$ in comparison groups, moderation analyses were not conducted for proportion of pain stimuli per trial or personal relevance of pain stimuli. Due to the number of attentional bias variables and moderators, we only report significant results in text. All moderator results are reported in Table 3.

\section{Attentional Bias Paradigm}

There was a significant effect for studies using free viewing tasks on duration of first fixation to pain-related stimuli compared to neutral stimuli $(\mathrm{k}=6, g=-0.36,95 \% \mathrm{Cl}-0.62:-0.10, p=0.007)$, but not for studies using the dot probe $(\mathrm{k}=14, g=0.007,95 \% \mathrm{Cl}-0.16: 0.18, p=0.94)$. This difference was significant $\left(Q_{1}=5.28, p=0.02\right)$. This indicates that free-viewing tasks detected when participants spending longer looking at pain rather than neutral stimuli on their first fixation, but this effect was not detected by the dot-probe task. Similarly, free-viewing tasks detected participants fixating more times on neutral relative to pain stimuli $(\mathrm{k}=10, g=-0.33,95 \% \mathrm{Cl}-0.70:-0.04, p=0.047)$ but the dot probe task $\operatorname{did} \operatorname{not}(\mathrm{k}=8, g=0.22,95 \% \mathrm{Cl}-0.18: 0.62, p=0.29)$, and this difference was again significant $\left(\mathrm{Q}_{1}=3.86\right.$, $p=0.049)$. A similar effect was found for dwell time where free-viewing tasks were more likely to detect participants spending a longer overall time looking at pain stimuli $(\mathrm{k}=9, g=0.75,95 \% \mathrm{Cl} 0.25: 1.24, p=$ 0.003) compared to the dot probe $(\mathrm{k}=15, g=0.24,95 \% \mathrm{Cl}-0.70: 0.04, p=0.22)$, but for overall dwell time the difference was not significant $\left(Q_{1}=2.5, p=0.11\right)$.

\section{Stimulus Modality}

There was a significant moderator effect on probability of first fixation and dwell time, such that there was a significant effect demonstrating vigilance for pain-related pictures (first fixation: $k=13, g=$ 0.70, 95\% Cl 0.39: 1.02, $p<.001$, dwell time: $\mathrm{k}=13, g=0.74,95 \% \mathrm{Cl} 0.36: 1.11, p<.001$ ) but not for painrelated words (first fixation: $\mathrm{k}=8, g=-0.003,95 \% \mathrm{Cl}-0.40: 0.40, p=0.99$, dwell time: $\mathrm{k}=12, g=0.11$, 
$95 \% \mathrm{Cl}-0.28: 0.50, p=0.59)$ respectively. That is, participants were more likely and spent longer time looking at pain pictures relative to neutral pictures, however, this effect was not found for pain-related words. This difference was significant in both cases (first fixation: $Q_{1}=7.31 p=0.007$, dwell time: $Q_{1}=$ $5.19, p=0.023)$. Additionally, there was a significant effect for pain-related pictures compared to neutral pictures on latency to first fixation, showing faster first fixations for pain pictures $(k=13, g=-0.36,95 \%$ $\mathrm{Cl}-0.71: 0.002, p=0.048)$ but not for words $(\mathrm{k}=8, g=-0.04,95 \% \mathrm{Cl}-0.48: 0.39, p=0.84)$, although this difference did not reach significance $\left(Q_{1}=1.19, p=0.28\right)$.

\section{Stimulus Presentation Time}

Presentation time had a significant moderating effect on duration of first fixation, such that there was a significant effect showing shorter first fixations on pain-related stimuli for presentations $>2000 \mathrm{~ms}(\mathrm{k}=4, g=-0.53,95 \% \mathrm{Cl}-0.85:-0.20, p=0.002)$ but not for presentations $\leq 2000 \mathrm{~ms}(\mathrm{k}=17, g=$ $0.03,95 \% \mathrm{Cl}-0.13: 0.19, p=0.72)$. This difference was significant $\left(Q_{1}=8.89, p=0.003\right)$. For number of fixations, there was a significant positive effect for presentations $\leq 2000$ ms indicating more fixations on pain-related stimuli $(\mathrm{k}=10, g=0.43,95 \% \mathrm{Cl} 0.12: 0.75, p=0.008)$ whereas for presentations $>2000 \mathrm{~ms}$ there was a significant negative effect indicating fewer fixations on pain-related stimuli $(k=8, g=-0.73$, $95 \% \mathrm{Cl}=1.09:-0.37, p<.001)$, and this difference was significant $\left(Q_{1}=22.37, p<.001\right)$. For probability of first fixation and dwell time there was a significant effect for presentations $\leq 2000 \mathrm{~ms}$ where participants were more likely to fixate on pain than neutral stimuli and did so for longer overall (First fixation: $k=15$, $g=0.44,95 \% \mathrm{Cl} 0.10: 0.77, p=0.01$ ), (Dwell time: $\mathrm{k}=18, g=0.48,95 \% \mathrm{Cl} 0.14: 0.82, p=0.006$ ), but not for $>2000 \mathrm{~ms}$ (first fixation: $\mathrm{k}=4, g=0.24,95 \% \mathrm{Cl}-0.41: 0.90, p=0.46$ and dwell time: $\mathrm{k}=7, g=0.33$, $95 \% \mathrm{Cl}-0.22: 0.87, p=0.24)$ respectively. However, this difference did not reach significance in either case $\left(Q_{1}=0.87, p=0.65\right.$ and $Q_{1}=0.21, p=0.65$, respectively $)$. 


\section{Fixation Definition}

In terms of definition of fixations, while there were no significant between group differences, the overall effect sizes indicating vigilance for probability of first fixation $(\mathrm{k}=16, g=0.43,95 \% \mathrm{Cl} 0.11$ : $0.76, p=0.009)$ and increased overall attention on total dwell time $(\mathrm{k}=22, g=0.48,95 \% \mathrm{Cl} 0.17: 0.79, p$ $=0.002$ ) were significant for fixations $\geq 100 \mathrm{~ms}$. Interestingly, these same patterns did not emerge when fixations were defined as $\geq 60 \mathrm{~ms}(\mathrm{k}=5, g=0.43,95 \% \mathrm{Cl}-0.16: 1.01, p=0.15$ and $\mathrm{k}=3, g=0.10,95 \% \mathrm{Cl}$ 0.73: $0.94, p=0.81$, respectively).

\section{Proportion of Pain Trials}

A significant moderating effect was found for proportion of pain trials on dwell time, such that participants showed a bias towards pain-related material in studies where $\leq 50 \%$ of trials were painrelated trials $(\mathrm{k}=13, \mathrm{~g}=0.76,95 \% \mathrm{Cl} 0.38: 1.14, \mathrm{p}<.001)$ but not where $>50 \%$ of total trials were pain trials $(\mathrm{k}=12, \mathrm{~g}=0.08,95 \% \mathrm{Cl}-0.32: 0.48, \mathrm{p}=0.70)$, and this difference was significant $(\mathrm{Q} 1=5.91, \mathrm{p}=$ 0.02). This pattern was the same for probability of first fixation where participants were more likely to focus on the pain-related compared to neutral stimuli when fewer than $50 \%$ of trials were pain-related $(\mathrm{k}=10, g=0.60,95 \% \mathrm{Cl} 0.22: 0.99, p=0.002)$, but not when more than $50 \%$ of trials were pain-related trials $(\mathrm{k}=11, g=0.27,95 \% \mathrm{Cl}-0.10: 0.65, p=0.15)$, but the difference did not reach significance $\left(\mathrm{Q}_{1}=\right.$ $1.44, p=0.23)$.

\section{Publication Year and Risk of Bias}

We investigated publication year and risk of bias as continuous moderators within a single metaregression. The overall meta-regression model was significant for latency to first fixation $\left(F_{2,20}=12.41\right.$, $\mathrm{p}<.01)$, with publication year $(r=0.30,95 \% \mathrm{Cl} 0.13: 0.48, \mathrm{p}<.001)$ and risk of bias $(r=-0.38,95 \% \mathrm{Cl}-0.67$ : $-0.08, p=0.01$ ) both independently accounting for variance in attentional bias. Results indicate more 
recent publications and those publications with less risk of bias are associated with smaller effect sizes. Additionally, risk of bias was a significant predictor in the dwell time $(r=-0.24,95 \% \mathrm{Cl}-0.46:-0.02, \mathrm{p}=$ $0.04)$ and number of fixation $(r=0.3395 \% \mathrm{Cl} 0.02: 0.63, \mathrm{p}=0.04)$ models, and in both cases less bias was associated with smaller effects.

\section{Summary}

Our moderator analyses did find that parameters were associated with whether attentional biases were found for pain-related words. On a number of variables, biases were observed on the free viewing task, but not the dot-probe. Similarly, in terms of stimuli, more biases were observed for painful facial expressions than for other stimuli. However, the only difference between people with and without chronic pain was observed for the number of fixations on sensory pain words. Biases were evident on some indices where the fixation definition was $100 \mathrm{~ms}$, rather than $60 \mathrm{~ms}$, when fewer than $50 \%$ of trials had pain-related stimuli but not when $>50 \%$ of trials did. Finally, presentation time appeared to change the pattern of attention, whereby in shorter presentation times participants had fewer, shorter fixations. In contrast, for longer presentation times, shorter first fixations on pain compared to neutral stimuli was observed. Finally, there was some evidence of smaller effect sizes on a number of parameters with later publications, and publications with higher quality ratings. This suggests that the current effect sizes could be inflated. 
Table 3 Series: Moderator Analyses

Table 3.1 Attentional Bias Paradigm as a Moderator

\begin{tabular}{|c|c|c|c|c|c|c|}
\hline Variable & $k$ & $n$ & Hedges' g & $95 \% \mathrm{Cl}$ & $\mathrm{I}^{2}$ & $\mathrm{Q}_{\mathrm{B}}$ \\
\hline \multicolumn{7}{|l|}{ Paradigm } \\
\hline Probability First Fixation & 18 & 728 & & & & 0.0 \\
\hline Dot Probe & 11 & 510 & 0.37 & -0.03 to 0.77 & $98.32 * * *$ & \\
\hline Free Viewing & 7 & 218 & 0.38 & -0.13 to 0.88 & $97.39 * * *$ & \\
\hline Latency to First Fixation & 19 & 792 & & & & 1.14 \\
\hline Dot Probe & 10 & 417 & -0.003 & -0.38 to 0.37 & 11.23 & \\
\hline Free Viewing & 9 & 375 & -0.31 & -0.72 to 0.11 & $96.98 * * *$ & \\
\hline Duration First Fixation & 20 & 668 & & & & $5.28 *$ \\
\hline Dot Probe & 14 & 500 & 0.007 & -0.16 to 0.18 & $79.01 * * *$ & \\
\hline Free Viewing & 6 & 168 & $-0.36 * *$ & -0.62 to -0.10 & $97.14 * * *$ & \\
\hline Average Fixation Duration & 14 & 424 & & & & 1.67 \\
\hline Dot Probe & 7 & 237 & 0.37 & -0.03 to 0.77 & $76.84 * * *$ & \\
\hline Free Viewing & 7 & 187 & 0.38 & -0.13 to 0.88 & $88.94 * * *$ & \\
\hline Dwell & 24 & 1140 & & & & 2.5 \\
\hline Dot Probe & 15 & 667 & 0.24 & -0.14 to 0.62 & $98.73 * * *$ & \\
\hline Free Viewing & 9 & 473 & $0.75^{* *}$ & 0.25 to 1.24 & $99.69 * * *$ & \\
\hline Number of Fixations & 18 & 624 & & & & $3.86^{*}$ \\
\hline Dot Probe & 8 & 341 & 0.22 & -0.18 to 0.62 & $84.70 * * *$ & \\
\hline Free Viewing & 10 & 283 & $-0.33^{*}$ & -0.70 to -0.04 & $95.46 * * *$ & \\
\hline
\end{tabular}


Table 3.2 Stimulus Type as a Moderator

\begin{tabular}{|c|c|c|c|c|c|c|}
\hline Stimulus type & $k$ & $n$ & Hedges' g & $95 \% \mathrm{Cl}$ & $\mathrm{I}^{2}$ & $\mathrm{Q}_{B}$ \\
\hline Probability First Fixation & 21 & 728 & & & & $7.31^{* *}$ \\
\hline Pictures & 13 & 397 & $0.70 * * *$ & 0.39 to 1.02 & $98.04 * * *$ & \\
\hline Words & 8 & 331 & -0.003 & -0.40 to 0.40 & $91.55^{* * *}$ & \\
\hline Latency to First Fixation & 21 & 792 & & & & 1.19 \\
\hline Pictures & 13 & 465 & $-0.36^{*}$ & -0.71 to -0.002 & $96.16 * * *$ & \\
\hline Words & 8 & 372 & -0.04 & -0.48 to 0.39 & 0.0 & \\
\hline Duration of First Fixation & 21 & 668 & & & & 0.76 \\
\hline Pictures & 11 & 293 & -0.14 & -0.35 to 0.07 & $96.24 * * *$ & \\
\hline Words & 10 & 375 & -0.005 & -0.22 to 0.21 & $78.61 * * *$ & \\
\hline Average Fixation Duration & 14 & 424 & & & & 1.5 \\
\hline Pictures & 9 & 222 & 0.19 & -0.02 to 0.40 & $90.12^{* * *}$ & \\
\hline Words & 5 & 202 & -0.03 & -0.30 to 0.25 & 0.0 & \\
\hline Dwell & 25 & 1140 & & & & $5.19 *$ \\
\hline Pictures & 13 & 652 & $0.74 * * *$ & 0.36 to 1.11 & $99.61 * * *$ & \\
\hline Words & 12 & 488 & 0.11 & -0.28 to 0.50 & $95.54 * * *$ & \\
\hline Number of Fixations & 18 & 624 & & & & 2.63 \\
\hline Pictures & 12 & 373 & -0.26 & -0.62 to 0.11 & $95.86 * * *$ & \\
\hline Words & 6 & 251 & 0.25 & -0.25 to 0.75 & $70.46 * * *$ & \\
\hline
\end{tabular}


Table 3.3 Fixation Definition as a Moderator

\begin{tabular}{|c|c|c|c|c|c|c|}
\hline Fixation definition & $k$ & $n$ & Hedges' g & $95 \% \mathrm{Cl}$ & $1^{2}$ & $\mathrm{Q}_{\mathrm{B}}$ \\
\hline Probability First Fixation & 21 & 728 & & & & 0.0 \\
\hline$\geq 100 \mathrm{~ms}$ & 16 & 633 & $0.43 * *$ & 0.11 to 0.76 & $98.19 * * *$ & \\
\hline$\geq 60 \mathrm{~ms}$ & 5 & 95 & 0.43 & -0.16 to 1.01 & $95.86^{* * *}$ & \\
\hline Latency to First Fixation & 21 & 792 & & & & 0.05 \\
\hline$\geq 100 \mathrm{~ms}$ & 16 & 688 & -0.25 & -0.57 to 0.07 & $94.16^{* * *}$ & \\
\hline$\geq 60 \mathrm{~ms}$ & 5 & 104 & -0.18 & -0.74 to 0.38 & $93.83 * * *$ & \\
\hline Duration of First Fixation & 21 & 668 & & & & 0.001 \\
\hline$\geq 100 \mathrm{~ms}$ & 18 & 573 & -0.08 & -0.24 to 0.09 & $94.36 * * *$ & \\
\hline$\geq 60 \mathrm{~ms}$ & 3 & 95 & -0.07 & -0.47 to 0.33 & 64.52 & \\
\hline Average Fixation Duration & 14 & 424 & & & & 0.05 \\
\hline$\geq 100 \mathrm{~ms}$ & 8 & 210 & 0.13 & -0.12 to 0.37 & $91.26 * * *$ & \\
\hline$\geq 60 \mathrm{~ms}$ & 6 & 214 & 0.08 & -0.19 to 0.35 & $66.43^{*}$ & \\
\hline Dwell & 25 & 1140 & & & & 0.70 \\
\hline$\geq 100 \mathrm{~ms}$ & 22 & 978 & $0.48^{* *}$ & 0.17 to 0.79 & $99.42 * * *$ & \\
\hline$\geq 60 \mathrm{~ms}$ & 3 & 162 & 0.10 & -0.73 to 0.94 & $97.85^{* * *}$ & \\
\hline Number of Fixations & 18 & 624 & & & & 0.0 \\
\hline$\geq 100 \mathrm{~ms}$ & 11 & 361 & -0.08 & -0.48 to 0.31 & $96.13^{* * *}$ & \\
\hline$\geq 60 \mathrm{~ms}$ & 7 & 263 & -0.08 & -0.57 to 0.40 & $87.97 * * *$ & \\
\hline
\end{tabular}


Table 3.4 Presentation Time as a Moderator

\begin{tabular}{|c|c|c|c|c|c|c|}
\hline Presentation Time & $k$ & $n$ & Hedges' g & $95 \% \mathrm{Cl}$ & $1^{2}$ & $\mathrm{Q}_{B}$ \\
\hline Probability First Fixation & 19 & 728 & & & & 0.87 \\
\hline$\leq 2000$ & 15 & 596 & $0.44^{*}$ & 0.10 to 0.77 & $98.31 * * *$ & \\
\hline$>2000$ & 4 & 132 & 0.24 & -0.41 to 0.90 & $94.79 * * *$ & \\
\hline Latency to First Fixation & 19 & 792 & & & & 2.19 \\
\hline$\leq 2000$ & 12 & 455 & 0.01 & -0.33 to 0.35 & 16.92 & \\
\hline$>2000$ & 7 & 337 & -0.43 & -0.89 to 0.04 & $97.65^{* * *}$ & \\
\hline Duration of First Fixation & 21 & 668 & & & & $8.89 * *$ \\
\hline$\leq 2000$ & 17 & 538 & 0.03 & -0.13 to 0.19 & $85.49 * * *$ & \\
\hline$>2000$ & 4 & 130 & $-0.53^{* *}$ & -0.85 to -0.20 & $98.12^{* * *}$ & \\
\hline Average Fixation Duration & 14 & 424 & & & & 0.02 \\
\hline$\leq 2000$ & 10 & 323 & 0.10 & -0.11 to 0.31 & $89.28^{* * *}$ & \\
\hline$>2000$ & 4 & 101 & 0.12 & -0.21 to 0.46 & $76.40 * * *$ & \\
\hline Dwell & 25 & 1140 & & & & 0.21 \\
\hline$\leq 2000$ & 18 & 771 & $0.48^{* *}$ & 0.14 to 0.82 & $99.05^{* * *}$ & \\
\hline$>2000$ & 7 & 369 & 0.33 & -0.22 to 0.87 & $99.70 * * *$ & \\
\hline Number of Fixations & 18 & 624 & & & & $22.37^{* * *}$ \\
\hline$\leq 2000$ & 10 & 379 & $0.43^{* *}$ & 0.11 to 0.75 & $89.33 * * *$ & \\
\hline$>2000$ & 8 & 245 & $-0.73 * * *$ & -1.09 to -0.37 & $93.12^{* * *}$ & \\
\hline
\end{tabular}


Table 3.5 Proportion of Pain Trials as a Moderator

\begin{tabular}{|c|c|c|c|c|c|c|}
\hline Proportion of pain trials & $k$ & $n$ & Hedges' g & $95 \% \mathrm{Cl}$ & $1^{2}$ & $\mathrm{Q}_{B}$ \\
\hline Probability First Fixation & 21 & 728 & & & & 1.44 \\
\hline$\leq 50 \%$ & 10 & 362 & $0.60 * *$ & 0.22 to 0.99 & $98.50 * * *$ & \\
\hline$>50 \%$ & 11 & 366 & 0.27 & -0.10 to 0.65 & $95.34 * * *$ & \\
\hline Latency to First Fixation & 21 & 792 & & & & 0.67 \\
\hline$\leq 50 \%$ & 8 & 363 & -0.37 & -0.79 to 0.06 & $94.42 * * *$ & \\
\hline$>50 \%$ & 13 & 429 & -0.14 & -0.48 to 0.20 & $92.86 * * *$ & \\
\hline Duration of First Fixation & 21 & 668 & & & & 1.43 \\
\hline$\leq 50 \%$ & 6 & 225 & 0.07 & -0.21 to 0.34 & $88.58^{* * *}$ & \\
\hline$>50 \%$ & 15 & 443 & -0.13 & -0.31 to 0.04 & $94.38 * * *$ & \\
\hline Average Fixation Duration & 14 & 424 & & & & 1.12 \\
\hline$\leq 50 \%$ & 3 & 161 & 0.28 & -0.09 to 0.66 & $95.74 * * *$ & \\
\hline$>50 \%$ & 11 & 263 & 0.05 & -0.15 to 0.26 & $77.57^{* * *}$ & \\
\hline Dwell & 25 & 1140 & & & & $5.91 *$ \\
\hline$\leq 50 \%$ & 13 & 719 & $0.76^{* * *}$ & 0.38 to 1.14 & $99.49 * * *$ & \\
\hline$>50 \%$ & 12 & 421 & 0.08 & -0.32 to 0.48 & $98.93 * * *$ & \\
\hline Number of Fixations & 18 & 624 & & & & 1.49 \\
\hline$\leq 50 \%$ & 5 & 252 & 0.20 & -0.34 to 0.74 & $90.65 * * *$ & \\
\hline$>50 \%$ & 13 & 372 & -0.20 & -0.54 to 0.15 & $94.77 * * *$ & \\
\hline
\end{tabular}

Note. Analyses here include a combination of chronic pain and healthy control participants.

$k$ values are the number of effect sizes; Hedges' $g$ values are the standardized differences of attentional biases between pain and neutral stimuli (small effect $=0.1-0.5$, medium effect $=0.5-0.8$, and large effect $>0.8$ ); $\mathrm{Cl}=$ confidence interval; $I^{2}$ values represent the within group heterogeneity statistic; $Q_{B}$ values represent the between group difference statistic.

${ }^{*} p<.05 ;{ }^{* *} p<.01 ; * * * p<.001$ 


\section{Discussion}

The current meta-analysis investigated the nature and time course of attentional biases to painrelated stimuli in studies using eye-tracking methodology in participants with and without chronic pain. Findings differ significantly from previous reviews focusing on reaction times, that all found evidence of attentional bias, at least to sensory pain words, in people with chronic pain compared to those without pain $[11 ; 47 ; 57]$. Our results suggest that when using eye-tracking methodologies, there is no compelling evidence that attentional biases are specific to people with chronic pain. Rather, they appear to be ubiquitous and that all participants, regardless of pain status, demonstrate attentional biases toward pain-related stimuli characterized by both biases in vigilance and sustained attention.

Ubiquitous biases in vigilance and sustained attention towards pain is consistent with an evolutionary account of attentional biases, suggesting that pain capturing and holding attention is adaptive in situations where one can quickly identify pain and apply a protective response in order to prevent potentially negative outcomes $[60 ; 66 ; 70]$. An earlier evolutionary account [71] concluded humans are hard-wired to consistently and readily distinguish painful facial expressions in other people because effective communication of pain activates aid from others, facilitating recovery and healing. This may explain why our results, that synthesized a larger portion of studies with facial stimuli, found people regardless of pain status were more likely to fixate on and spend longer gazing at painful facial expressions. Importantly, the same biases can be adaptive in one context but not another [66]. Empirical evidence supports this notion that motivation and context influence attentional biases [39; 50]. These ideas are not necessarily at odds with current models of chronic pain. For example, the misdirected problem-solving model [17] proposes that prioritization of pain and entrapment in a hopeless and ineffective cycle to 'solve' the pain impedes on other life goals. Similarly, the fear avoidance model [70] suggests it is the priority to control pain rather than prioritizing a valued life that 
lead attentional biases towards pain become associated with disability and avoidance. Therefore, the context in which attentional biases occur is crucial [66].

Our results identified important moderators that should be considered in future research. Selection of stimuli appears critical for attentional bias research, with results indicating observed biases in early and sustained attention were more evident for pictures than word stimuli for all participants. The increase in more ecologically valid facial stimuli in eye-tracking paradigms may explain the universality of attentional biases to pain, since previous analyses that found attentional biases to be specific for people with chronic pain included mostly sensory pain words [11; 57]. Interestingly, our only result that revealed a greater bias for people with chronic pain compared to those without was for probability of first fixation for painful facial expressions. This result should be interpreted with caution as it is based only on three studies and it is worth noting that there were only two studies $(n=75)$ in the meta-analysis that used sensory word stimuli with a chronic pain population. Hence, these results are preliminary and may differ in different contexts.

Several other task parameters affected results. For example, we found that pictures (faces in particular) more reliably led to attentional biases, and conceptually it has been argued that pictures are more ecologically valid than words [71]. Similarly, fixations of only $60 \mathrm{msec}$ did not give rise to attentional biases. The shorter fixations are, the more likely they are to be artefactual $[21 ; 22]$ and hence we would expect to see more effects with longer fixation durations, as was the case. Free-viewing style tasks that do not require a response (unlike the dot-probe task) are more naturalistic and gave rise in this meta-analysis to attentional biases more often, and finally having fewer than $50 \%$ of trials being pain-related likely reduces habituation to stimuli giving rise to more robust results. Hence, in future studies that use existing paradigms to increase their ecological validity and reliability, we make the following recommendations: use of gaze contingent trials, use of picture rather than word stimuli, 
selection of free-viewing style tasks with eye-tracking, inclusion of $\leq 50 \%$ pain-related trials, fixation definitions of $\geq 100 \mathrm{~ms}$ and contemporaneous measurement of theoretically relevant variables.

Our findings also point towards the contextual role of threat in attentional biases to pain. Under conditions of low threat, participants more quickly oriented towards pain-related words and spent more time focusing on pain-related than neutral words. In contrast, under high threat conditions, participants made fewer fixations on pain-related words. While this was a large effect, it was based on only three studies and the confidence intervals were very large. It is also notable that the difference between high and low threat conditions was only significant for number of fixations. Therefore, these results need to be interpreted cautiously, but do confirm the importance of investigating context, such as the threatening nature of impending pain. In addition, we found little evidence of individual differences in attentional biases according to participants' levels of pain catastrophizing or pain-related fear, and there were not enough studies to consider the role of attentional control. Consistent with the wider literature, we acknowledge that gaze direction will be influenced by both emotional and cognitive processes and thus, despite current findings, individual differences in emotional and cognitive factors will still be important to consider in future studies. The interaction between perceived threat and individual differences in emotional factors will be important to consider, as will the interaction between attentional control and developmental stage.

The risk of bias analysis was generally consistent with the internal and external ratings in previous meta-analyses [11;57], despite $17 / 24$ studies that were not included in the earlier reviews. Accounting for task engagement scored significantly lower than any other criterion (33\%), with the rest scoring over $60 \%$. The use of eye-tracking technology is aimed at ensuring eye gaze is focused on the task and measuring task engagement. However, after repetition of multiple trials eye-tracking data may become less accurate due to "drift" [23] and therefore the reliability of gaze data can be increased by 
regular drift checks throughout the task [13]. One example is making the presentation of each trial contingent on fixation on the central cross e.g.[48; 51]. This is particularly important with free viewing tasks that do not require participant responses. There also appears to be a significant impact of study quality, with more recent publications and higher quality studies being associated with smaller effects for several attentional bias variables. This suggests that as research designs improve the attentional bias outcomes might be smaller, but they are also likely to be more consistent.

Based on the findings discussed above, and consistent with recent reviews $[57 ; 61 ; 66]$, we recommend a shift in paradigm in attentional bias research. Future studies should aim to include active goal pursuit and manipulation of context to capture the functional and contextual nature of attentional biases in pain. Existing examples include adapting attentional bias tasks so participant responses impact a non-pain goal, such as winning or losing money [49]; or using virtual reality to simulate daily tasks such as bending over or reaching [62]. It will also be important to consider these motivational and contextual factors within the context of interventions that target pain-related attention. For example, in mindfulness-based therapies for chronic pain, patients are often directed to increase their attention towards their body (which can include painful body sites) but to do so with elevated acceptance and non-judgmental awareness. In other words, patients learn to change the quality of their attention from anxious vigilance towards accepting awareness. Indeed, one study has already demonstrated that attentional processes (based on gaze behavior) moderate the efficacy of mindfulness, such that those who can readily disengage from pain stimuli (shorter duration of first fixation) benefit more from mindfulness [52]. Eye-tracking paradigms administered before and after such treatments, particularly using stimuli that capture the threat value of pain, could be informative (see also [66]). Additionally, research on 'action observation', commonly studied in the context of Parkinson's Disease and stroke, could also be usefully applied. For example, these studies have shown that guiding patients' attention towards pictures of goal-directed movements can promote motor rehabilitation via activation of the 
motor cortex [2]. Similar strategies could be used to guide patients' attention towards pictorial stimuli representing feared movements in graded exposure therapies. This would again help reveal how attentional processes influence pain-directed behavior and can be harnessed for intervention. Finally, when current theories highlight the role of vigilance in chronic pain, they refer to vigilance to pain sensations rather than vigilance to representations of pain. Future research should investigate attentional biases towards actual pain, which may include examining attentional biases using signals of impending pain e.g.[9; 33; 64; 65], body locations affected by pain e.g.[59], ambiguous somatosensory stimuli e.g.[37] and real, experienced pain e.g.[38].

This meta-analysis has limitations that point towards more future research directions. Firstly, there were relatively few studies that included between-group analyses, which means that some analyses are statistically underpowered. Further, in cases where between-group analyses could be conducted, the confidence intervals were broad, potentially obscuring group differences. Similarly, planned moderators could not be assessed adequately with the chronic pain group alone, and so these relationships may differ from those reported. Furthermore, while we examined the associations between self-report measures such as pain catastrophising and fear of pain, fewer than $30 \%$ of studies reported these outcomes.

Despite these limitations, we provide a comprehensive synthesis of extant eye-tracking studies that reveals the nature and time course of attention biases in pain. In summary, when using more direct measurements of attention (gaze behavior), we failed to replicate the (small) attentional biases observed in patients with chronic pain relative to healthy controls in reaction time paradigms. Instead, our results suggest that, at least for pictorial or linguistic stimuli representative of pain, attentional biases to pain are ubiquitous and not specific to people with chronic pain. That is, biases indicative of both vigilance towards pain and difficulty disengaging from pain were observed for all participants regardless of the pain status. Future research requires design paradigms that assess biases to actual 
somatosensory stimuli, especially across various contexts so that the contexts in which attentional processes contribute towards pain can be better understood.

\section{Acknowledgements:}

Dimitri Van Ryckeghem is supported by the FNR fund C18/BM/12671141.

Emma Jones is supported by a Research Training Program (RTP) Domestic Scholarship.

Emma Jones: No conflict

Louise Sharpe: No conflict

Sally Andrews: No conflict

Ben Colagiuri: No conflict

Joanne Dudeney: No conflict

Elaine Fox: No conflict

Lauren Heathcote: No conflict

Jennifer Lau: No conflict

Jemma Todd: No conflict

Stefaan Van Damme: No conflict

Dimitri Van Ryckeghem: No conflict

Tine Vervoort: No conflict 


\section{References}

[1] Comprehensive Meta Analysis (Version 3.3.070). Inglewood, NJ, USA: Biostat Inc., 2014.

[2] Abbruzzese G, Avanzino L, Marchese R, Pelosin E. Action observation and motor imagery: innovative cognitive tools in the rehabilitation of Parkinson's disease. Parkinson's Disease 2015;2015.

[3] Aldrich S, Eccleston C, Crombez G. Worrying about chronic pain: vigilance to threat and misdirected problem solving. Behaviour research and therapy 2000;38(5):457-470.

[4] Bar-Haim Y, Lamy D, Pergamin L, Bakermans-Kranenburg MJ, Van ljzendoorn MH. Threat-related attentional bias in anxious and nonanxious individuals: a meta-analytic study. Psychological bulletin 2007;133(1):1.

[5] Begg CB, Mazumdar M. Operating characteristics of a rank correlation test for publication bias. Biometrics 1994:1088-1101.

[6] Borenstein M, Hedges LV, Higgins JP, Rothstein HR. A basic introduction to fixed-effect and randomeffects models for meta-analysis. Research synthesis methods 2010;1(2):97-111.

[7] Borenstein M, Hedges LV, Higgins JP, Rothstein HR. Introduction to meta-analysis: John Wiley \& Sons, 2011.

[8] Borenstein M, Higgins JP, Hedges LV, Rothstein HR. Basics of meta-analysis: 12 is not an absolute measure of heterogeneity. Research synthesis methods 2017;8(1):5-18.

[9] Clauwaert A, Torta DM, Danneels L, Van Damme S. Attentional modulation of somatosensory processing during the anticipation of movements accompanying pain: an event-related potential study. The Journal of Pain 2018;19(2):219-227.

[10] Cohen J. Statistical power analysis for the behavioral sciences: Routledge, 2013.

[11] Crombez G, Van Ryckeghem DM, Eccleston C, Van Damme S. Attentional bias to pain-related information: a meta-analysis. Pain 2013;154(4):497-510.

[12] Crombez G, Vlaeyen JW, Heuts PH, Lysens R. Pain-related fear is more disabling than pain itself: evidence on the role of pain-related fear in chronic back pain disability. Pain 1999;80(1-2):329339.

[13] Dalrymple KA, Manner MD, Harmelink KA, Teska EP, Elison JT. An Examination of Recording Accuracy and Precision From Eye Tracking Data From Toddlerhood to Adulthood. Frontiers in Psychology 2018;9(803).

[14] Dear BF, Sharpe L, Nicholas MK, Refshauge K. The psychometric properties of the dot-probe paradigm when used in pain-related attentional bias research. The Journal of Pain 2011;12(12):1247-1254.

[15] Duc AH, Bays P, Husain M. Eye movements as a probe of attention. Progress in brain research, Vol. 171: Elsevier, 2008. pp. 403-411.

[16] Duval S, Tweedie R. Trim and fill: a simple funnel-plot-based method of testing and adjusting for publication bias in meta-analysis. Biometrics 2000;56(2):455-463.

[17] Eccleston C, Crombez G. Worry and chronic pain: a misdirected problem solving model. Pain 2007;132(3):233-236.

[18] Egger M, Smith GD, Schneider M, Minder C. Bias in meta-analysis detected by a simple, graphical test. Bmj 1997;315(7109):629-634.

[19] Fashler SR, Katz J. More than meets the eye: visual attention biases in individuals reporting chronic pain. Journal of pain research 2014;7:557.

[20] Fashler SR, Katz J. Keeping an eye on pain: investigating visual attention biases in individuals with chronic pain using eye-tracking methodology. Journal of pain research 2016;9:551.

[21] Fischer B, Ramsperger E. Human express saccades: extremely short reaction times of goal directed eye movements. Experimental Brain Research 1984;57(1):191-195. 
[22] Fischer B, Weber H. Express saccades and visual attention. Behavioral and Brain Sciences 1993;16(3):553-567.

[23] Frank MC, Vul E, Saxe R. Measuring the development of social attention using free-viewing. Infancy 2012;17(4):355-375.

[24] Franklin ZC, Holmes PS, Fowler NE. Eye gaze markers indicate visual attention to threatening images in individuals with chronic back pain. Journal of clinical medicine 2019;8(1):31.

[25] Heathcote L, Lau J, Mueller S, Eccleston C, Fox E, Bosmans M, Vervoort T. Child attention to pain and pain tolerance are dependent upon anxiety and attention control: An eye-tracking study. European journal of pain 2017;21(2):250-263.

[26] Hedges LV, Olkin I. Statistical methods for meta-analysis: Academic press, 2014.

[27] Higgins JP, Thompson SG. Quantifying heterogeneity in a meta-analysis. Statistics in medicine 2002;21(11):1539-1558.

[28] IASP. Classification of chronic pain: Descriptions of chronic pain syndromes and definitions of pain terms (2nd ed.). In: IAftso Pain editor. Seattle: IASP Press, 1994.

[29] Jackson T, Su L, Wang Y. Effects of higher versus lower threat contexts on pain-related visual attention biases: an eye-tracking study of chronic pain. The Journal of Pain 2018;19(6):649-659.

[30] Jackson T, Yang Z, Su L. Pain-related gaze biases and later functioning among adults with chronic pain: a longitudinal eye-tracking study. Pain 2019;160(10):2221-2228.

[31] Kaseweter K, Rose K, Bednarik S, Woodworth M. More than meets the eye: the role of psychopathic traits in attention to distress. Psychology, Crime \& Law 2020;26(2):109-127.

[32] Lee J, Kim S, Shin S, Wachholtz A, Lee J. Attentional Engagement for Pain-Related Information among Individuals with Chronic Pain: The Role of Pain Catastrophizing. Pain Research and Management 2018;2018.

[33] Ling Y, Yang Z, Jackson T. Visual attention to pain cues for impending touch versus impending pain: An eye tracking study. European Journal of Pain 2019;23(8):1527-1537.

[34] Liossi C, Schoth DE, Godwin HJ, Liversedge SP. Using eye movements to investigate selective attention in chronic daily headache. PAIN ${ }^{\circledR}$ 2014;155(3):503-510.

[35] Mahmoodi-Aghdam M, Dehghani M, Ahmadi M, Banaraki AK, Khatibi A. Chronic pain and selective attention to pain arousing daily activity pictures: Evidence from an eye tracking study. Basic and clinical neuroscience 2017;8(6):467.

[36] Mazidi M, Dehghani M, Sharpe L, Dolatshahi B, Ranjbar S, Khatibi A. Time course of attentional bias to painful facial expressions and the moderating role of attentional control: an eye-tracking study. British Journal of Pain 2019:2049463719866877.

[37] Moseley GL, Arntz A. The context of a noxious stimulus affects the pain it evokes. PAIN ${ }^{\circledR}$ 2007;133(1-3):64-71.

[38] Noel M, Rabbitts JA, Fales J, Chorney J, Palermo TM. The influence of pain memories on children's and adolescents' post-surgical pain experience: A longitudinal dyadic analysis. Health Psychology 2017;36(10):987.

[39] Notebaert L, Crombez G, Vogt J, De Houwer J, Van Damme S, Theeuwes J. Attempts to control pain prioritize attention towards signals of pain: An experimental study. Pain 2011;152(5):1068-1073.

[40] Parsons S, Kruijt A-W, Fox E. Psychological Science Needs a Standard Practice of Reporting the Reliability of Cognitive-Behavioral Measurements. Advances in Methods and Practices in Psychological Science 2019;2(4):378-395.

[41] Peters ML, Vieler JS, Lautenbacher S. Dispositional and induced optimism lead to attentional preference for faces displaying positive emotions: An eye-tracker study. The Journal of Positive Psychology 2016;11(3):258-269.

[42] Pincus T, Morley S. Cognitive-processing bias in chronic pain: a review and integration. Psychological bulletin 2001;127(5):599. 
[43] Priebe J, Messingschlager M, Lautenbacher S. Gaze behaviour when monitoring pain faces: An eyetracking study. European Journal of Pain 2015;19(6):817-825.

[44] Roelofs J, Peters ML, Zeegers MP, Vlaeyen JW. The modified Stroop paradigm as a measure of selective attention towards pain-related stimuli among chronic pain patients: a meta-analysis. European Journal of Pain 2002;6(4):273-281.

[45] Rosenthal R. The file drawer problem and tolerance for null results. Psychological bulletin 1979;86(3):638.

[46] Schoth DE, Godwin HJ, Liversedge SP, Liossi C. Eye movements during visual search for emotional faces in individuals with chronic headache. European Journal of Pain 2015;19(5):722-732.

[47] Schoth DE, Nunes VD, Liossi C. Attentional bias towards pain-related information in chronic pain; a meta-analysis of visual-probe investigations. Clinical psychology review 2012;32(1):13-25.

[48] Schoth DE, Wu J, Zhang J, Guo X, Liossi C. Eye-movement behaviours when viewing real-world painrelated images. European Journal of Pain 2019;23(5):945-956.

[49] Schrooten MG, Van Damme S, Crombez G, Kindermans H, Vlaeyen JW. Winning or not losing? The impact of non-pain goal focus on attentional bias to learned pain signals. Scandinavian journal of pain 2018;18(4):675-686.

[50] Schrooten MG, Van Damme S, Crombez G, Peters ML, Vogt J, Vlaeyen JW. Nonpain goal pursuit inhibits attentional bias to pain. Pain 2012;153(6):1180-1186.

[51] Sharpe L, Brookes M, Jones E, Gittins C, Wufong E, Nicholas M. Threat and fear of pain induces attentional bias to pain words: An eye-tracking study. European Journal of Pain 2017;21(2):385396.

[52] Shires A, Sharpe L, Newton John TR. The relative efficacy of mindfulness versus distraction: The moderating role of attentional bias. European Journal of Pain 2019;23(4):727-738.

[53] Skinner IW, Hübscher M, Moseley GL, Lee H, Wand BM, Traeger AC, Gustin SM, McAuley JH. The reliability of eyetracking to assess attentional bias to threatening words in healthy individuals. Behavior research methods 2018;50(5):1778-1792.

[54] Sun ZK, Wang JY, Luo F. Experimental pain induces attentional bias that is modified by enhanced motivation: An eye tracking study. European Journal of Pain 2016;20(8):1266-1277.

[55] Todd J, Sharpe L, Colagiuri B, Khatibi A. The effect of threat on cognitive biases and pain outcomes: An eye-tracking study. European Journal Of Pain (London, England) 2016;20(8):1357-1368.

[56] Todd J, Sharpe L, Johnson A, Perry KN, Colagiuri B, Dear BF. Towards a new model of attentional biases in the development, maintenance, and management of pain. Pain 2015;156(9):15891600.

[57] Todd J, van Ryckeghem DM, Sharpe L, Crombez G. Attentional bias to pain-related information: a meta-analysis of dot-probe studies. Health psychology review 2018;12(4):419-436.

[58] Van Bockstaele B, Lamens L, Salemink E, Wiers RW, Bögels SM, Nikolaou K. Reliability and validity of measures of attentional bias towards threat in unselected student samples: seek, but will you find? Cognition and Emotion 2020;34(2):217-228.

[59] Van Damme S, Bulcke CV, Van Den Berghe L, Poppe L, Crombez G. Do patients with chronic unilateral orofacial pain due to a temporomandibular disorder show increased attending to somatosensory input at the painful side of the jaw? PeerJ 2018;6:e4310.

[60] Van Damme S, Legrain V, Vogt J, Crombez G. Keeping pain in mind: a motivational account of attention to pain. Neuroscience \& Biobehavioral Reviews 2010;34(2):204-213.

[61] Van Ryckeghem D, Crombez G. Pain and attention: towards a motivational account. 2018.

[62] Van Ryckeghem DM. The interference of pain with task performance: Increasing ecological validity in research. Scandinavian journal of pain 2017;16(1):91-92.

[63] Van Ryckeghem DM, Crombez G. Attentional bias and chronic pain: Where to go from here? Pain 2014;155(1):6-7. 
[64] Van Ryckeghem DM, Crombez G, Goubert L, De Houwer J, Onraedt T, Van Damme S. The predictive value of attentional bias towards pain-related information in chronic pain patients: a diary study. PAIN ${ }^{\circledR} 2013 ; 154(3): 468-475$.

[65] Van Ryckeghem DM, Crombez G, Van Hulle L, Van Damme S. Attentional bias towards pain-related information diminishes the efficacy of distraction. PAIN ${ }^{\circledR} 2012 ; 153(12): 2345-2351$.

[66] Van Ryckeghem DM, Noel M, Sharpe L, Pincus T, Van Damme S. Cognitive biases in pain: an integrated functional-contextual framework. Pain 2019;160(7):1489-1493.

[67] Vervoort T, Trost Z, Prkachin KM, Mueller SC. Attentional processing of other's facial display of pain: An eye tracking study. PAIN ${ }^{\circledR} 2013 ; 154(6): 836-844$.

[68] Vervoort T, Trost Z, Sütterlin S, Caes L, Moors A. Emotion regulatory function of parent attention to child pain and associated implications for parental pain control behaviour. PAIN ${ }^{\circledR}$ 2014;155(8):1453-1463.

[69] Vlaeyen JW, Linton SJ. Fear-avoidance and its consequences in chronic musculoskeletal pain: a state of the art. Pain 2000;85(3):317-332.

[70] Vlaeyen JW, Morley S, Crombez G. The experimental analysis of the interruptive, interfering, and identity-distorting effects of chronic pain. Behaviour research and therapy 2016;86:23-34.

[71] Williams ACdC. Facial expression of pain: an evolutionary account. Behavioral and brain sciences 2002;25(4):439-455.

[72] Yang Z, Jackson T, Chen H. Effects of chronic pain and pain-related fear on orienting and maintenance of attention: an eye movement study. The Journal of Pain 2013;14(10):1148-1157.

[73] Yang Z, Jackson T, Gao X, Chen H. Identifying selective visual attention biases related to fear of pain by tracking eye movements within a dot-probe paradigm. PAIN ${ }^{\circledR} 2012 ; 153(8): 1742-1748$. 